\title{
Relationships between insulin resistance, blood glucose levels and gastric motility : an electrogastrography and external ultrasonography study
}

\author{
Masako Kaji, Masahiro Nomura*, Yoshiyuki Tamura ${ }^{\dagger}$, and Susumu Ito \\ Department of Digestive and Cardiovascular Medicine, Institute of Health Biosciences, The Univer- \\ sity of Tokushima Graduate School, *Faculty of Integrated Art and Sciences, Department of Human \\ and Social Sciences, The University of Tokushima; and ${ }^{\dagger}$ National Hospital Organization Zentsuji \\ National Hospital
}

\begin{abstract}
Background: Detailed information concerning the influence of insulin resistance on gastrointestinal motility are not available. Methods : The relationship between insulin resistance and gastric motility and emptying, and changes in gastric motility with changes in blood glucose level were investigated using electrogastrography (EGG) and external ultrasonography in 20 non-diabetic subjects. The homeostasis model assessment ratio (HOMA-R) was used as an index of insulin resistance. The cut off value for HOMA-R was set at 1.7. Subjects with HOMA-R $\geq 1.7$ were the high HOMA-R group, and HOMA-R < 1.7 were the normal HOMA-R group. In the EGG data, a Fast Fourier Transform (FFT) analysis was performed, and the mean peak power was compared among brady-, normal-, and tachy-gastria. Results : In the fasting state, the ratios of brady-gastria in EGG and HOMA-R were significantly positively correlated, and the ratios of normal-gastria and HOMA-R were significantly negatively correlated. When glucose was intravenously administered, the ratio of normal-gastria was significantly decreased and the ratio of bradygastria was significantly increased in subjects with a high HOMA-R. In a gastric emptying test by external ultrasonography, gastric emptying activity was significantly decreased in subjects with a high HOMA-R. Conclusions: We conclude that insulin resistance induces abnormal gastric motility. Though abnormal gastric motility is related to HOMA-R, the findings herein suggest that incretin, showing insulin resistance, or an adipocyokine might be related to the differences in EGG in both groups. The relation between abnormal gastric motility and other serum parameters (incretin, adipocyokine, etc) would contribute to a better understanding of this process. J. Med. Invest. 54 : 168-176, February, 2007
\end{abstract}

Keywords : gastric motility, gastric emptying, insulin resistance, electrogastrography, HOMA-R

\section{INTRODUCTION}

Insulin resistance was defined as a condition that

Received for publication November 27, 2006 ; accepted January 9, 2007.

Address correspondence and reprint requests to Masako Kaji, MD, Department of Digestive and Cardiovascular Medicine, Institute of Health Biosciences, The University of Tokushima Graduate School, Kuramoto-cho, Tokushima 770-8503, Japan and Fax : +81-88-633-9235. requires more insulin than the normal levels to exert various cell-, organ-, and individual-level insulin actions by Berson, et al. in 1970. The concept that insulin resistance is involved in the progression from a normal condition to abnormal glucose tolerance and the onset of type 2 diabetes has been established, and insulin resistance-associated hyperinsulinemia has been reported to have a marked influence on the development and course of arteriosclerotic diseases 
through obesity, hypertension, and hyperlipidemia (1) and enhanced sympathetic nervous activity (2).

Diabetic paralysis of gastric motility and delayed gastric emptying have been reported in cases of diabetic autonomic neuropathy (3-5), and their objective evaluation by EGG has been reported (6-13). However, there have been no detailed reports of the influence of insulin resistance on gastrointestinal motility. In the present study, we investigated the relationship between insulin resistance as a background factor of diabetes and gastric motility and emptying, and changes in gastric motility with changes in the blood glucose levels using EGG and external ultrasonography.

\section{MATERIAL AND METHODS}

\section{1) Subjects}

Twenty non-diabetic subjects (mean age : $53.0 \pm$ 18.0 years, 11 men and 9 women) were investigated. None of the subjects had a past medical history of digestive or circulatory disorders, and their physical findings were normal without an abnormality on standard 12-lead electrocardiography and chest X-ray radiography. Patients with hypertension and diabetes and subjects who had been treated with autonomic agonists, such as anticholinergics and $\beta$-blockers, within 1 month were excluded from the study. Malignant disease and liver disease were also excluded in the present study.

EGG was measured from a fasting state to 1 hour after an intravenous administration of a $5 \%$ glucose solution at a drip infusion rate of $300 \mathrm{ml} /$ hour. A gastric emptying test by external ultrasonography was also performed in the same subjects. Written informed consent was obtained from all subjects prior to the start of the tests.

\section{2) Insulin resistance}

For the insulin resistance index, the homeostasis model assessment ratio (HOMA-R) was calculated by the following equation $(14,15)$ :
HOMA-R $=$ Fasting glucose $(\mathrm{mg} / \mathrm{dl}) \times$ Fasting insulin $(\mu \mathrm{U} / \mathrm{ml}) \div 405$

In the present study, the cut off value for HOMA-R was set at 1.7. The subjects with HOMA-R $\geq 1.7$ were assigned to the high HOMA-R group, and HOMA-R $<1.7$ were assigned to the normal HOMA-R group. Table 1 compares fasting blood sugar (FBS), glycol hemoglobin A1c (HbA1c), body mass index (BMI) in high and normal HOMA-R groups. FBS and HbA1c were in the normal range in both the high and normal HOMA-R groups, and there was no significant difference in both groups. However, the BMI of the high HOMA-R group was significantly higher than normal HOMA-R group.

\section{3) EGG measurement}

A Nipro electrogastrograph, EG (A\&G, Tokyo) was used for the EGG. As shown in Figure 1, 4 surface probe electrodes ( $\mathrm{CH} 1$ - 4) were arranged so as to surround the stomach, and the central electrode $(\mathrm{N})$ was placed at the mid-point between the navel and xiphoid process. The EGG was recorded by a bipolar lead between the central and 4 surface probe electrodes. The sampling interval was 1 second, and the measurement frequency was 2.1 - 6.0 cycles/min (cpm). On data recording, the influence of respiration was completely eliminated using 10thorder filters. In addition, the influence of body movement was minimized using a linear phase filter to minimize the distortion of EGG signals.

\section{4) Analysis of EGG}

The recorded EGG data were input into a personal computer through $\mathrm{RC} 232 \mathrm{C}$, and analyzed using software exclusive for EG (NIPRO ESC1, A\&D, Tokyo). A Fast Fourier Transformation (FFT) was applied to 512 points, and the dominant frequencies and peak powers for the 4 channels were averaged. Frequencies of $2.4 \mathrm{cpm}$ or lower, 2.4-3.6 cpm, and $3.6 \mathrm{cpm}$ or higher were designated as brady-, normal-, and tachy-gastria, respectively, based on the EGG spectral analysis (FFT analysis), and their mean peak powers were compared.

Table 1 FBS, HbA1c, BMI in high and normal HOMA-R groups

\begin{tabular}{lccc}
\hline & normal HOMA-R group & high HOMA-R group & $p$ value \\
\hline FBS (mg/dl) & $83.4 \pm 5.7$ & $88.4 \pm 6.7$ & ns \\
HbA1c (\%) & $4.90 \pm 0.27$ & $4.68 \pm 0.83$ & ns \\
BMI & $21.3 \pm 3.3$ & $25.9 \pm 8.9$ & $p<0.05$ \\
\hline
\end{tabular}

FBS, fasting blood sugar; HbA1c, glycol hemoglobin A1c; BMI, body mass index; HOMA-R, homeostasis model assessment ratio ; mean \pm standard deviation 


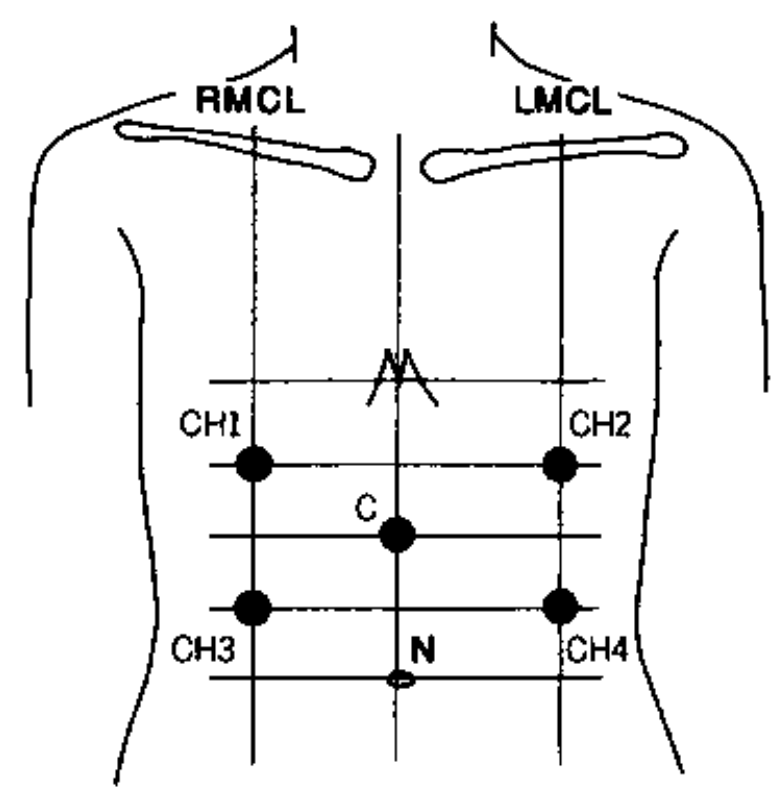

Figure 1 Positions of the EGG recording electrodes RMCL, right mid-clavicular line ; LMCL, left mid-clavicular line ; $\mathrm{N}$, navel; $\mathrm{CH} 1$, channel 1 ; $\mathrm{CH} 2$, channel 2 ; $\mathrm{CH} 3$, channel 3 ; $\mathrm{CH} 4$, channel 4 ; C, central terminal electrode.

5) Gastric emptying test by external ultrasonography

The antral stomach area was measured 1 and 15 minutes after the ingestion of a test diet using an external ultrasonic diagnostic system (Aplio SSA770A, Toshiba, Tokyo), and the rate of change (\%) was evaluated. In a morning fasting state, an ultrasonic probe was placed at a position where the abdominal aorta, superior mesenteric artery, and cross section of the antral stomach could be seen in one view, and the cross-sectional area of the antral stomach was measured using calipers incorporated in the system. For the liquid test diet, $400 \mathrm{ml}$ of consommé soup $\left(37^{\circ} \mathrm{C}, 13 \mathrm{kcal}\right.$, Ajinomoto, Tokyo) was used.

\section{6) Statistical analysis}

All values are presented as the means \pm standard deviation. Statistical analyses were performed using a personal computer. Comparison among the each group was performed using the unpaired $t$ and paired t-test, and $p<0.05$ was regarded as significant. Linear logistic regression analysis was used to assess correlations between continuous variables ; the correlation coefficient $r$ was calculated. StatView 5.0 software (SAS Institute Inc., Cary, North Caroline, USA) was used for the statistical analyses.

\section{RESULTS}

\section{1) Analysis of fasting EGG waveforms and frequency}

Figure 2a shows an example of the EGG waveforms and the FFT analysis of their frequency in a non-insulin resistant subject with a normal HOMA-R (0.41). The EGG waveforms were regular sinusoidal waves, the peak frequency of the FFT analysis was $3.2 \mathrm{cpm}$ with a unimodal pattern, and the mean peak power of the 4 channels was about $75.0 \mu \mathrm{V}$.

There were 2 subjects with a high HOMA-R of
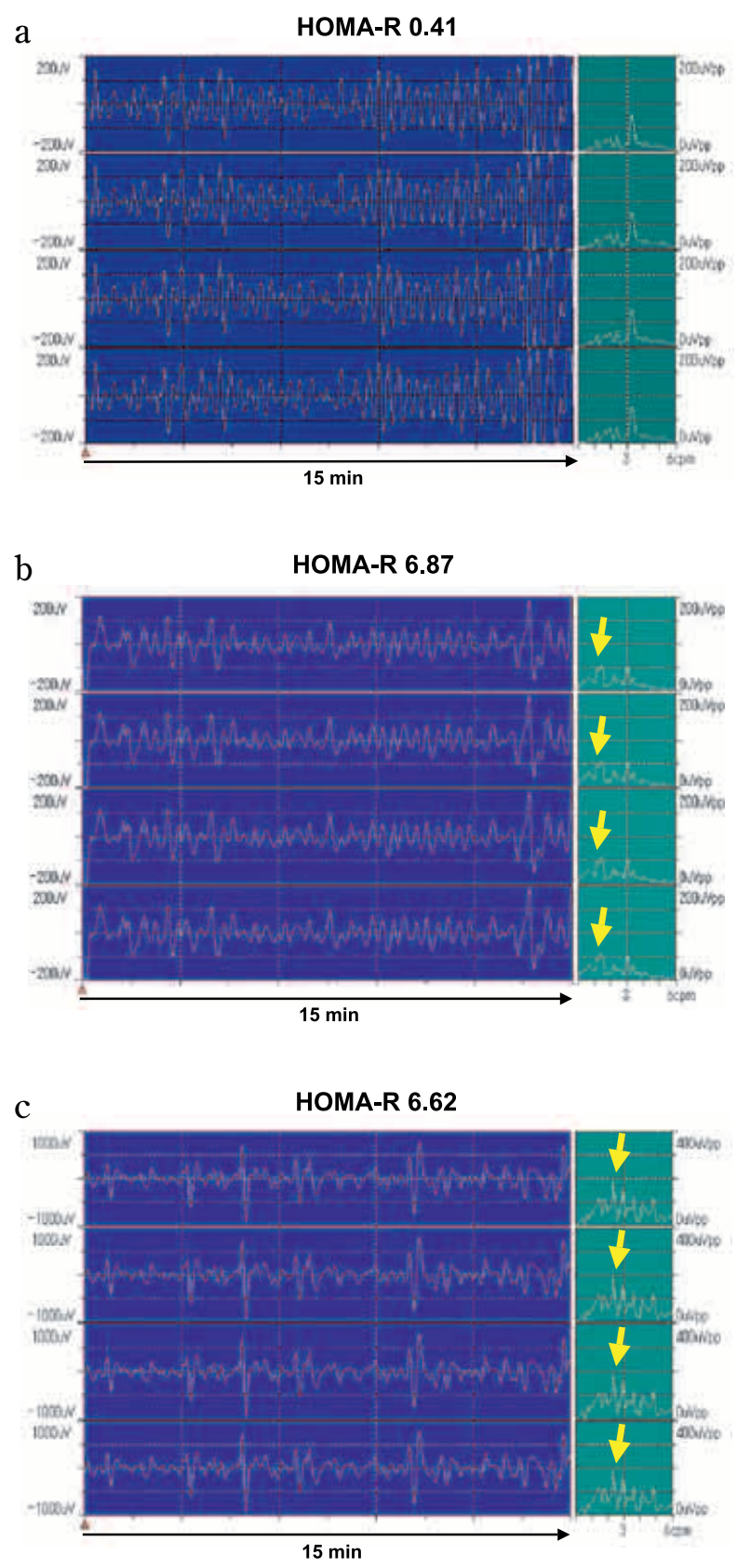

Figure 2 Examples of EGG waveforms and an FFT analysis of their frequencies

(a : a non-insulin resistant case, b : a case with a HOMA-R of 6.87 suggesting insulin resistance, $\mathrm{c}:$ a case with a HOMA-R of 6.62 suggesting insulin resistance) 
more than 6.0. These two cases were not diabetic patients, and one was a 50 year old male (FBS $83 \mathrm{mg}$ / dl, IRI $33.5 \mu \mathrm{U} / \mathrm{ml}$, HOMA-R 6.87, HbA1c 4.7\%) and other was a 43 year old male (FBS $106 \mathrm{mg} / \mathrm{dl}$, IRI $25.3 \mu \mathrm{U} / \mathrm{ml}$, HOMA-R 6.62, HbA1c $5.3 \%$ ).

Figure $2 \mathrm{~b}$ shows an example of EGG waveforms and an FFT analysis of their frequency in a subject with a high HOMA-R (6.87), indicative of insulin resistance. The frequency of the EGG waveforms is lower than that in Figure 2a, and the peak was noted at a low frequency close to $1.5 \mathrm{cpm}$ on FFT analysis. The peak power decreased at about $3 \mathrm{cpm}$, showing a bimodal pattern.

Figure 2c shows an example of EGG waveforms and an FFT analysis of their frequency in another subject with a high HOMA-R (6.62) suggesting insulin resistance. The sinusoidal waveforms seen in Figure $2 \mathrm{a}$ are absent, and irregular waveforms can be seen. On FFT analysis, a low frequency of about $2.4 \mathrm{cpm}$ was dominant, but multimodal peaks were also noted at about $3-5 \mathrm{cpm}$.

2) Correlation between the HOMA-R and ratios of brady-, normal-, and tachy-gastria on fasting EGG

Figure 3 shows the correlation between the HOMA-R and the ratios of brady-, normal-, and tachygastria on a fasting EGG. A significant positive correlation was noted between the ratio of brady-gastria on EGG and HOMA-R ( $\mathrm{r}=+0.469, \mathrm{p}=0.040)$. In contrast, a significant negative correlation was noted between the ratio of normal-gastria and HOMA-R $(r=-0.534, p=0.015)$. However, no significant correlation was noted between the ratio of tachy-gastria and HOMA-R.

3) Analysis of EGG waveforms and frequency before and after intravenous glucose administration
Figure 4a shows an example of EGG waveforms and an FFT analysis of their frequency before and after glucose administration in a subject with a normal HOMA-R (0.73). Before glucose administration, as seen in the subject shown in Figure 3a, the dominant frequency was about $3 \mathrm{cpm}$. After intravenous glucose administration, the amplitude of the EGG was generally lowered, and the peak power value was decreased on FFT analysis, but the dominant frequency was still noted at about $3 \mathrm{cpm}$, as before the administration.

Figure $4 \mathrm{~b}$ shows an example of EGG waveforms and an FFT analysis of their frequency in a subject with a high HOMA-R (4.01) suggesting insulin resistance. Before glucose administration, multimodal divided peaks were noted within a range of 1-4 $\mathrm{cpm}$. After intravenous glucose administration, the power value of about the 3-cpm waveforms was slightly decreased, but no significant change was noted in the peak power of brady-gastria with a frequency of $2.4 \mathrm{cpm}$ or less.

4) Comparison of the ratios of brady-, normal-, and tachy-gastria before and after intravenous glucose administration between subjects with normal and high HOMA-R

Figure 5 shows a comparison of the ratios of brady-, normal-, and tachy-gastria before and after intravenous glucose administration for subjects with normal and high HOMA-R. In the subjects with a normal HOMA-R, the ratio of each waveform was not significantly changed by glucose administration. In contrast, in subjects with a high HOMA-R, intravenous glucose administration significantly decreased the ratio of normal-gastria $(\mathrm{p}<0.05)$ and increased the ratio of brady-gastria $(p<0.05)$. However, no significant change was noted in the ratio of tachy-gastria.

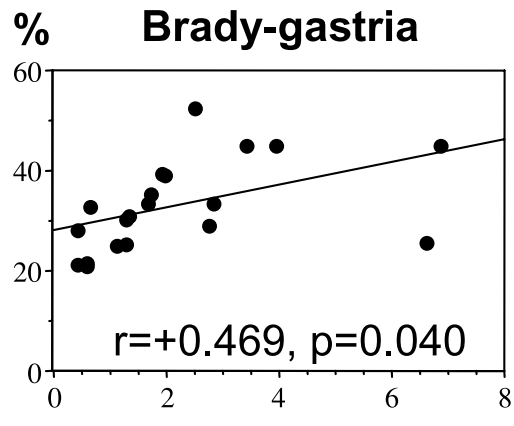

HOMA-R
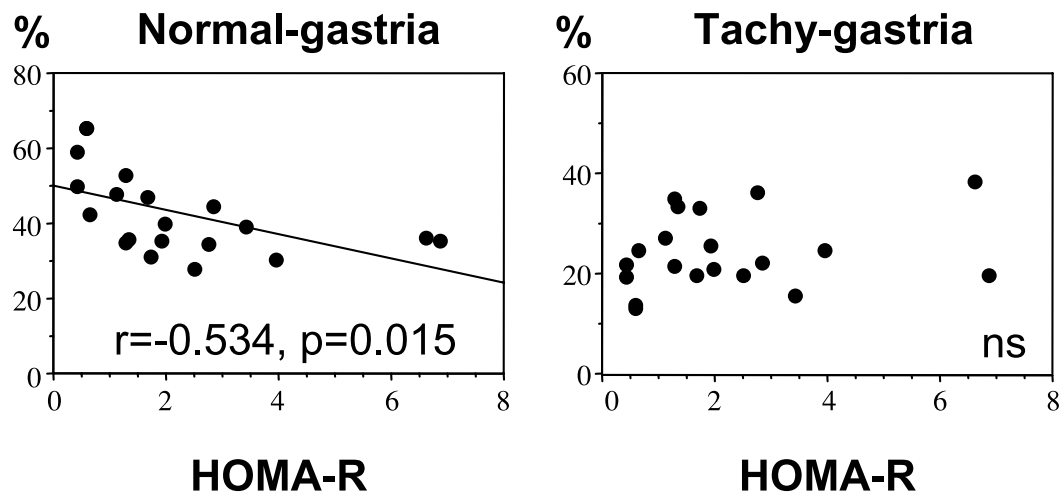

Figure 3 Correlation between HOMA-R and the ratios of brady-, normal-, and tachy-gastria on a fasting EGG 


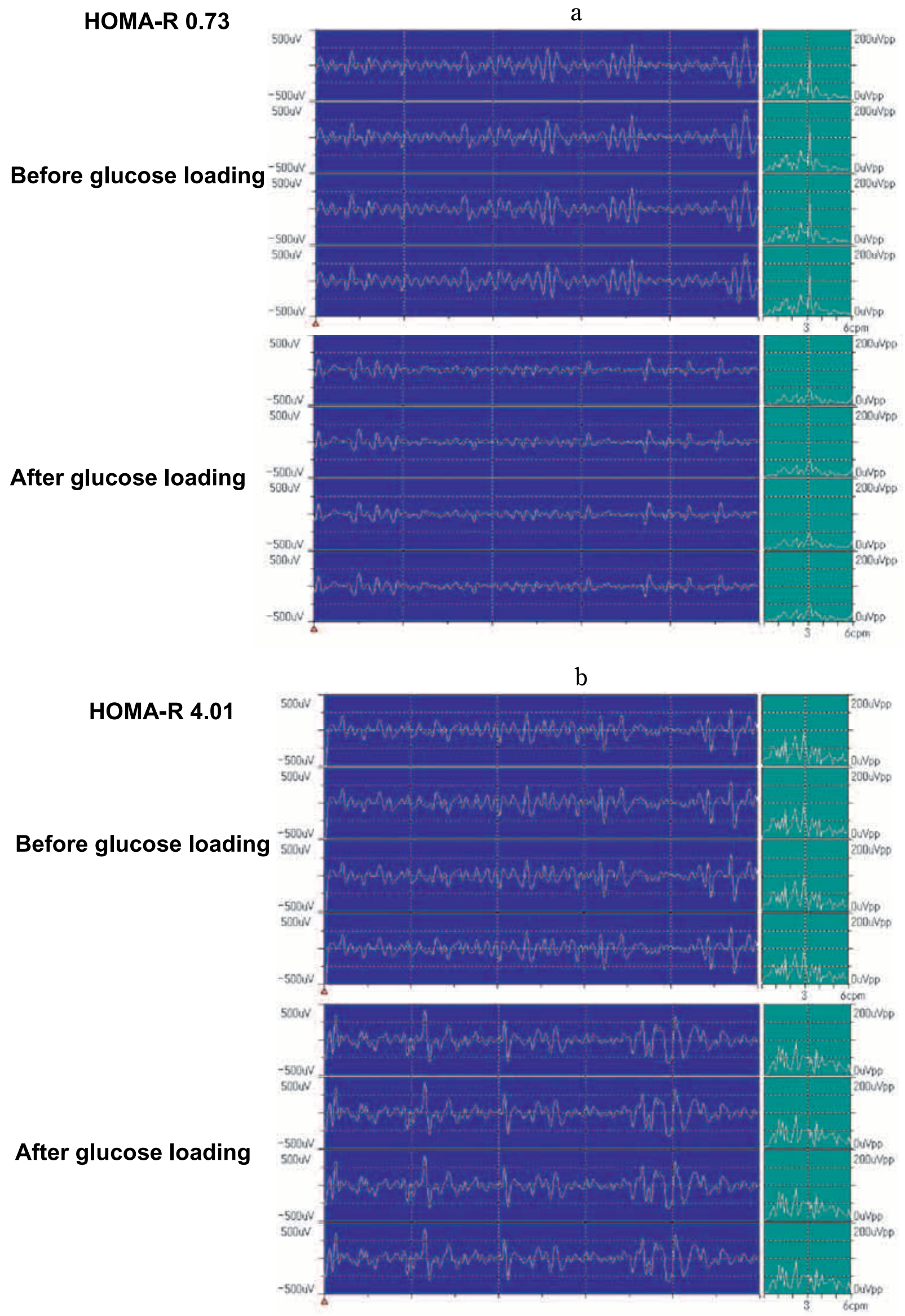

Figure 4 Examples of EGG waveforms and an FFT analysis of their frequencies before and after glucose loading (a : a non-insulin resistant case with a HOMA-R of 0.73 , b : a case with a HOMA-R of 4.01 suggesting insulin resistance) 

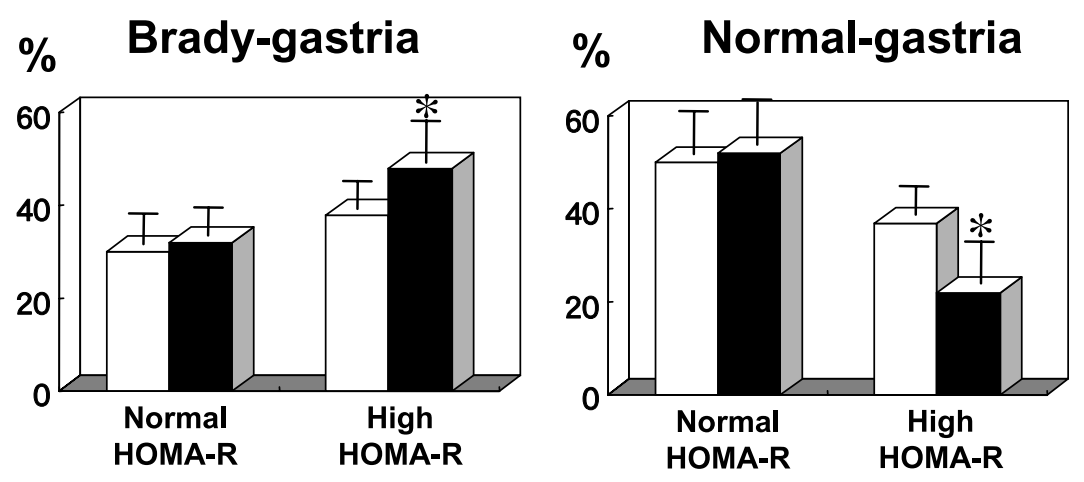

$\% \quad$ Tachy-gastria

* $p<0.05$ vs Before glucose loading

Before glucose load

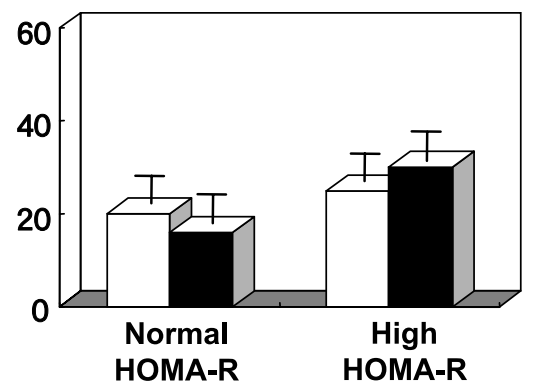

After glucose loading

Figure 5 Ratios of brady-, normal-, and tachy-gastria before and after intravenous glucose administration in subjects with normal and high HOMA-R

\section{5) Gastric emptying test by external ultrasonogra- phy}

Figure 6a shows an example of a gastric emptying test by external ultrasonography in a subject with a normal HOMA-R (0.41). The rate of change in the cross-sectional antral gastric area was $55.9 \%$. Figure $6 \mathrm{~b}$ shows the test results for a subject with a high HOMA-R (6.62) showing insulin resistance. The rate of change in the antral gastric sectional area was $18.9 \%$, lower than that in non-insulin resistant

a

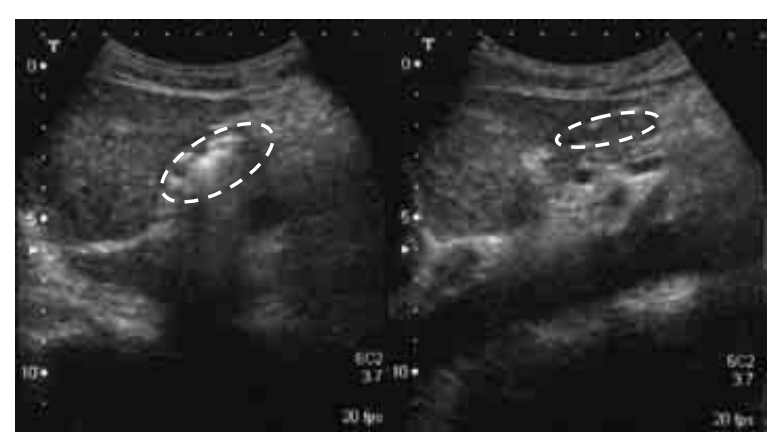

b

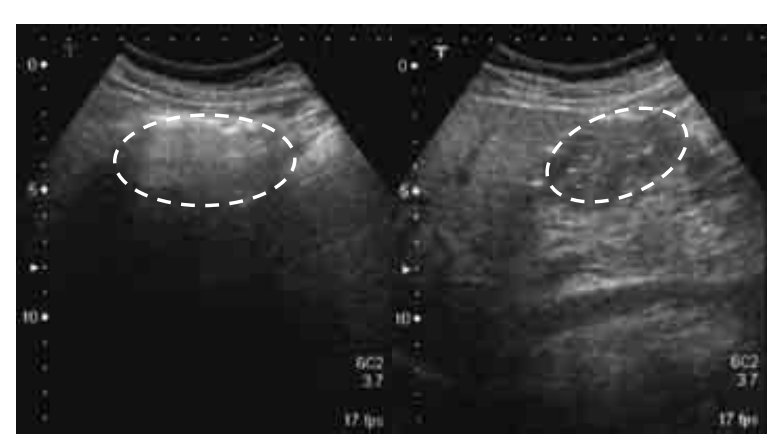

Figure 6 Examples of gastric emptying tests by external ultrasonography

(a : a non-insulin resistant case with a HOMA-R of $0.41, \mathrm{~b}:$ a case with a HOMA-R of 6.62 suggesting insulin resistance) subjects.

The rates of change in the antral gastric sectional area in subjects with normal and high HOMA-R were $58.6 \pm 6.6 \%$ and $25.8 \pm 18.9 \%$, respectively, showing a significant decrease in the case of insulinresistant subjects $(\mathrm{p}<0.05)$.

\section{DISCUSSION}

Regarding the relationship between insulin resistance-associated hyperinsulinemia and gastrointestinal motility, 3-cpm waveforms, which are considered to represent normal gastric movement, were decreased, and brady-gastria, which represents abnormal gastric movement, was increased in subjects suggested to have insulin resistance. An intravenous glucose load increased the abnormal waveforms representing brady-gastria on EGG in subjects with insulin resistance. Moreover, gastric motility was decreased in an external ultrasonographic gastric emptying test in subjects with insulin resistance, suggesting an association between insulin resistance and gastric motility.

In the present study, the differences in fasting EGG between the normal and high HOMA-R groups cannot be explained by both blood glucose level and hyperinsulinemia, because the EGG spectrum did not change after an intravenous administration of glucose in normal HOMR-R subjects. Although the spectral power of the EGG was correlated with HOMA-R, the findings suggest that incretin, showing insulin resistance, or an adipocyokine might be related to the EGG differences in both groups. Further information on the relation between abnormal 
gastric motility and other serum parameters (incretin, adipocyokine, etc) would clarify this issue.

\section{1) Insulin resistance and $E G G$ findings}

EGG is a non-invasive gastric motility examination that records the electrical action potential of gastric smooth muscle through the body surface. EGG was initially reported by Alvarez, et al. in 1922 (16), and subsequent developments and advancement of digital filters have allowed more stable EGG measurements $(17,18)$.

Gastric electrical activity is generated by a pacemaker located at a site in the upper $1 / 3$ of the stomach body on the greater curvature side, and is classified into electrical control activity (ECA), which is generated at about $3 \mathrm{cpm}$ and is transmitted toward the pyloric side, and electrical response activity (ERA), which is involved in rhythmical contractions of the stomach (19-21). EGG is generally considered to reflect both ECA and ERA : the cycle of gastric movement is consistent with the EGG cycle, and the strength of gastric motility is generally considered to be related to the peak power on EGG (22).

On a frequency analysis of EGG, waveforms with a normal periodic variation of $2.4-3.6 \mathrm{cpm}$ were designated as normal-gastria, fast periodic waveforms with a frequency of $3.6 \mathrm{cpm}$ or higher as tachygastria, and slow periodic waveforms with a frequency of $2.4 \mathrm{cpm}$ or less as brady-gastria. In healthy subjects, regular periodic waveforms at about $3 \mathrm{cpm}$, which indicate normal-gastria, are noted in both the fasting and postprandial states on EGG, and the peak power increases with an enhancement in gastric peristalsis. An increase in the ratio of normal-gastria is considered to correspond to an increase in gastric peristalsis, i,e., an increase in the action potential of gastric smooth muscle, and the rate of change in peak power within this frequency range on EGG is correlated with gastric contraction (11).

In our investigation of insulin resistance and gastric motility, the dominant frequency was about $3 \mathrm{cpm}$ in the fasting state in subjects with a normal HOMA-R, as in generally healthy individuals. In subjects with a high HOMA-R, the peak frequency was low, or peaks were noted at high and low frequencies, showing a multimodal pattern, while the peak power of the about-3cpm waveforms was decreased. Regarding the relationship between HOMA-R and the ratio of the waveforms with each frequency, an increase in HOMA-R was significantly negatively correlated with the ratio for normal-gastria, and significantly positively correlated with the ratio for brady- gastria. These findings suggested that insulin resistance is associated with decreased gastric motility.

Regarding changes in EGG with an elevation in blood glucose levels after intravenous glucose administration, an elevation in blood glucose levels led to a decreased peak power and ratio of normal-gastria in subjects with a normal HOMA-R, suggesting that gastric contractility was inhibited. In subjects with a high HOMA-R, indicative of insulin resistance, in addition to a decrease in the peak power of normalgastria, the ratio of brady-gastria was increased with an elevation in blood glucose levels, indicating abnormal gastric motility, and decreased gastric contractility due to hyperinsulinemia-induced abnormal autonomic nervous function.

\section{2) Insulin resistance and gastric emptying activity}

The gastric emptying test using external ultrasonography has been increasingly used as a simple non-invasive method (23-25). The rate of change in the sectional area of the antral stomach was lower in subjects with a high HOMA-R than in subjects with a normal HOMA-R. Thus, this method also suggests that insulin resistance reduces gastric emptying activity.

\section{3) Relationship between insulin resistance and gas- tric motility}

EGG and external ultrasonographic investigations indicate a reduced gastric motility in the presence of insulin resistance. Considering that insulin resistance is a background factor for type 2 diabetes, the abnormal autonomic nervous function may have appeared by a certain mechanism as a prestep to diabetes.

A glucose tolerance test was not performed in the high HOMA-R cases in Figure 2b, c. Fasting blood sugar and HbA1c levels in these subjects were in the normal range, but impaired tolerance subjects might have been included in the HOMA-R group. The findings herein suggest that increased insulin resistance is related to a high BMI level in the high HOMA-R group. Quilliot, et al. (26) reported that autonomic nervous activity using heart rate variability was correlated to BMI and was independent of age and HOMA-R. The findings of the present study suggest that a high level of BMI in the high HOMA-R group is related to autonomic nervous abnormality.

It has recently been established that interstitial cells of Cajal adjacent to Auerbach's plexus manage gastric smooth muscle movement, serving as a gas- 
tric pacemaker system (27). This pacemaker system has been reported to contain neurotransmitter and hormone receptors, integrate information from these receptors, and control gastric movement. The gastrointestinal pacemaker system transmits neuromuscular and hormonal signals through periodic electrical activity (28). Studies of this pacemaker system may lead to a re-evaluation of abnormal gastrointestinal movement.

\section{STUDY LIMITATIONS}

The involvement of autonomic nerves and gastrointestinal hormones in insulin resistance-associated abnormal gastric motility is assumed, but the possibility that the pacemaker system integrates these actions remains to be investigated. An assessment of gastric motility by EGG may lead to the development of methods for evaluating gastrointestinal hormonal and autonomic nervous activities.

\section{REFERENCES}

1. DeFronzo RA, Ferrannini E: Insulin resistance. A multifaceted syndrome responsible for NIDDM, obesity, hypertension, dyslipidemia, and atherosclerotic cardiovascular disease. Diabetes Care 14 : 173-94, 1991

2. Shimamoto K, Hirata A, Fukuoka M, Higashiura K, Miyazaki Y, Shiiki M, Masuda A, Nakagawa $\mathrm{M}$, Iimura $\mathrm{O}$ : Insulin sensitivity and the effects of insulin on renal sodium handling and pressor systems in essential hypertensive patients. Hypertension 23(Suppl 1) : I29-33, 1994

3. Kassander P : Asymptomatic gastric retention in diabetics (gastroparesis diabeticorum). Ann Intern Med 48 : 797-812, 1958

4. Hornbuckle K, Barnett JL: The diagnosis and work-up of the patient with gastroparesis. J Clin Gastroenterol 30 : 117-24, 2000

5. Hoogerwerf WA, Pasricha PJ, Kalloo AN, Schuster MM : Pain : the overlooked symptom in gastroparesis. Am J Gastroenterol 94 : 102933, 1999

6. Rothstein RD, Alavi A, Reynolds JC : Electrogastrography in patients with gastroparesis and effect of long-term cisapride. Dig Dis Sci 38 : 1518-24, 1993

7. Koch KL: Electrogastrography : physiological basis and clinical application in diabetic gas- tropathy. Diabetes Technol Ther 3 : 51-62, 2001

8. Chen JD, Lin Z, Pan J, McCallum RW : Abnormal gastric myoelectrical activity and delayed gastric emptying in patients with symptoms suggestive of gastroparesis. Dig Dis Sci 41 : 1538-45, 1996

9. Jebbink HJ, Bruijs PP, Bravenboer B, Akkermans $\mathrm{LM}$, vanBerge-Henegouwen GP, Smout AJ : Gastric myoelectrical activity in patients with type I diabetes mellitus and autonomic neuropathy. Dig Dis Sci 39 : 2376-83, 1994

10. Soykan I, Lin Z, Sarosiek I, McCallum RW : Gastric myoelectrical activity, gastric emptying, and correlations with symptoms and fasting blood glucose levels in diabetic patients. Am J Med Sci 317 : 226-31, 1999

11. Torichigai N, Iwase M, Gotoh D, Uchizono $\mathrm{Y}$, Yoshinari M, Iida M : Association of abnormal electrogastrography and cardiac autonomic neuropathy in diabetic patients without upper gastrointestinal symptoms. J Japan Diab Soc 45 : 167-71, 2002

12. Obara $\mathrm{M}:$ Gatric dysfunction assessed by electrogastrography(EGG) and the acetaminophen absorption technique in diabetic patients. Jpn J Clin physiol 28 : 137-43, 1998

13. Okamoto H, Nomura M, Nakaya Y, Uehara K, Saito K, Kimura M, Chikamori K, Ito S : Effects of epalrestat, an aldose reductase inhibitor, on diabetic neuropathy and gastroparesis. Intern Med 42 : 655-64, 2003

14. Turner RC, Holman RR, Matthews D, Hockaday TD, Peto J : Insulin deficiency and insulin resistance interaction in diabetes : estimation of their relative contribution by feedback analysis from basal plasma insulin and glucose concentrations. Metabolism $28: 1086-96,1979$

15. Matthews DR, Hosker JP, Rudenski AS, Naylor BA : Treacher DF, Turner RC: Homeostasis model assessment : insulin resistance and betacell function from fasting plasma glucose and insulin concentrations in man. Diabetologia 28 : 412-9, 1985

16. Alvarez WC : The electrogastrogram and what it shows. JAMA $78: 1116-9,1922$

17. Chen JD, McCallum RW : Clinical applications of electrogastrography. Am J Gastroenterol 88 : 1324-36, 1993

18. Mintchev MP, Bowes KL: Computer simulation of the effect of changing abdominal thickness on the electrogastrogram. Med Eng Phys $20: 177-81,1998$ 
19. Smout AJ, van der Schee EJ, Grashuis JL : What is measured in electrogastrography? Dig Dis Sci 25 : 179-87, 1980

20. Familoni BO, Bowes KL, Kingma YJ, Cote KR: Can transcutaneous recordings detect gastric electrical abnormalities? Gut 32 : 141-6, 1991

21. Sarna SK : Gastrointestinal electrical activity : terminology. Gastroenterology 68 : 1631-5, 1975

22. Chen JD, Zou X, Lin X, Ouyang S, Liang J : Detection of gastric slow wave propagation from the cutaneous electrogastrogram. Am J Physiol 277(2 Pt 1) : G424-30, 1999

23. Bateman DN, Whittingham TA : Measurement of gastric emptying by real-time ultrasound. Gut 23 : 524-7, 1982

24. King PM, Adam RD, Pryde A, McDicken WN, Heading RC : Relationships of human antroduodenal motility and transpyloric fluid movement: non-invasive observations with real-time ultra- sound. Gut 25 : 1384-91, 1984

25. Holt S, McDicken WN, Anderson T, Stewart IC, Heading RC : Dynamic imaging of the stomach by real-time ultrasound - - a method for the study of gastric motility. Gut 21 : 597-601, 1980

26. Quilliot D, Fluckiger L, Zannad F, Drouin P, Ziegler O : Impaired autonomic control of heart rate and blood pressure in obesity : role of age and of insulin-resistance. Clin Auton Res 11 : 79-86, 2001

27. Ordog T, Takayama I, Cheung WKT, Ward SM, Sanders KM : Remodeling of networks of interstitial cells of Cajal in a murine model of diabetic gastroparesis. Diabetes $49: 1731-9$, 2000

28. Takayama I, Horiguchi K, Daigo Y, Mine T, Fujino MA, Ohno S : The interstitial cells of $\mathrm{Ca}-$ jal and a gastroenteric pacemaker system. Arch Histol Cytol 65 : 1-26, 2002 